\title{
INTÉRÊT ET MÉTHODOLOGIE DE L'ÉCHOGRAPHIE QUANTITATIVE
}

\author{
G. BERGER et P. LAUGIER \\ UA CNRS 1458 Imagerie Paramétrique, 15 rue de l'Ecole de Médecine, F-75006 Paris, France
}

\begin{abstract}
When processing the echographic image, the whole information contained in the echographic signal is not used. Part of this information related to the complex way in which ultrasound and biological tissue interact is lost through signal analysis. The goal of tissue characterization is the quantitative estimation of acoustic parameters or texture parameters by appropriate signal processing.of echographic data. One particularly important parameter which can be estimated is the ultrasonic attenuation and its frequency dependance. Attenuation has been successfully measured in various clinical fields as muscle, liver or breast pathologies.
\end{abstract}

\section{L'APPROCHE QUANTITATIVE}

\section{I.1. Position du problème}

L'importance de l'échographie dans le plateau technique de l'imagerie médicale s'est largement confirmée ces dernières années. Des progrès constants ont été effectués dans le but d'améliorer la résolution spatiale de l'image. On a vu apparâitre l'imagerie à hautefréquence, les techniques endoscopiques, les biopsies guidées, l'imagerie de flux, et plus récemment les images tridimensionnelles.

L'image échographique ne reflète qu'une partie de l'information contenue dans le signal échographique. Le contenu fréquentiel du signal est perdu lors de la réalisation de l'image. Considérons en effet le transducteur ultrasonore excité par une brève impulsion électrique et rayonnant une onde de pression ultrasonore dans le milieu de propagation. Une partie de l'énergie ultrasonore est réfléchie aux interfaces séparant deux tissus biologiques (par exemple: interface graisse muscle) ou diffusée par les structures de petite dimension en comparaison avec la longueur d'onde. C'est le même transducteur qui sert en réception pour la détection du signal ultrasonore aprés son interaction avec le milieu biologique. L'image échographique (B-scan) est réalisée à partir du signal redressé et filtré. Les seules informations conservées sont le temps de vol des échos ainsi que leur amplitude. Dans le cas des tissus biologiques où l'approximation de Born est vérifiée, il y a une correspondance univoque entre temps de vol d'un écho et la profondeur à laquelle on trouve la cible. Pour connaître la relation entre le temps de vol et la profondeur il faut avoir la valeur de la célérité des ultrasons dans les différents milieux traversés. On admet généralement que la célérité est constante dans tous les tissus mous traversés. L'amplitude des échos (déterminée par le coefficient de réflection à l'interface ou par la section efficace de rétrodiffusion des diffuseurs) module le niveau de gris affiché au pixel correspondant de l'image échographique. L'image échographique ainsi obtenue est une image de la réflectivité du tissu. Cette image est assez proche d'une image morphologique. Le choix de la fréquence d'émission du transducteur ultrasonore dépend de la profondeur de pénétration nécessaire pour une application clinique définie. La profondeur 
de pénétration dans les tissus diminue lorsque la fréquence augmente, et la résolution de l'image augmente. La fréquence varie de $3.5 \mathrm{MHz}$ a $10 \mathrm{MHz}$ environ pour la routine médicale. En mode échographique, le signal émis est de brève durée et son spectre en fréquence est large. Le contenu en fréquences du signal échographique se modifie au cours de la propagation (effet de diffraction) et de l'interaction (atténuation, diffusion et réflection) avec les tissus. L'image échographique ne représente que l'amplitude du signal reçu en fonction de la profondeur (Fig.1). Le contenu en fréquences du signal est alors perdu.

La caractérisation tissulaire a pour but une exploitation plus complète du signal échographique. Il est possible d'extraire des informations quantitatives que l'image morphologique (ou image échographique) ne représente pas et de corréler ces informations à l'état du tissu. La nature quantitative des informations extraites est également un avantage. Il s'agit d'un domaine qui évolue depuis plusieurs années. Les premiers travaux furent en général effectués "in vitro" sur des tissus excisés. Après un enthousiasme prématuré pour les applications "in vivo", il fallut approfondir les connaissances théoriques et expérimentales relatives à l'interaction des ultrasons avec les tissus. Ces étapes ont été progressivement franchies et, selon nous, nous mènent seulement à l'orée d'une échographie quantitatives et de ses applications cliniques. Il est possible de diviser l'échographie quantitative en deux domaines: l'analyse de texture et la l'estimation de paramètres acoustiques. Ces approches concernent dans la grande majorité des cas le signal radio-fréquence (r.f.) mais également en ce qui concerne certaines approches en analyse de texture le signal vidéo. Le signal échographique est numérisé à haute fréquence après amplification à la sortie du transducteur ultrasonore. Analysé avant la réalisation de l'image, ce signal conserve toutes ses informations potentielles.

\subsection{L'analyse de texture}

L'analyse de texture est la description statistique du signal échographique. Les tissus mous peuvent être modélisés comme un ensemble de diffuseurs de distribution spatiale aléatoire. Lorsque la sonde émet le signal incident, celui-ci atteint chaque diffuseur au bout d'un temps de propagation en relation avec la distance sonde-diffuseur. Chaque diffuseur se comporte alors comme une source secondaire émettant une onde sphérique. Si la densité des diffuseurs était faible et que les temps d'arrivée sur la sonde réceptrice de ces ondes soient séparés d'une durée du pulse, alors les différents diffuseurs seraient bien résolus en nombre et en distance (Fig. 2a). Ce n'est pas le cas des milieux biologiques où la densité des diffuseurs est élevée. Dans ce cas, les différentes ondes sphériques interfèrent à la surface du transducteur qui effectue en chaque point une sommation cohérente de la pression acoustique (Fig.2b). La contribution des diffuseurs au signal étant mélangée, leur nombre et leur localisation n'apparait pas clairement. Il suffit par exemple de changer l'angle sous lequel sont vus ces diffuseurs pour que le signal résultant soit modifié (Fig.2c). Dans la mesure où leur distribution est aléatoire (ce que vérifient particulièrement bien foie, thyroide, rate) le signal ultrasonore est une grandeur aléatoire. L'enveloppe du signal est également une grandeur aléatoire. Comme l' amplitude en chaque point de l'enveloppe module la brillance sur l'écran, des aspects plus ou moins brillants ne seront que le reflet d'interférences constructives où destructives. Ce schéma d'interférence, ou "speckle" est visible sur l'image échographique sous la forme d'un aspect granité. Les lois statistiques du premier et du deuxième ordre sont bien connues pourvu que soient vérifiées quelques hypothèses (expérimentalement, ces hypothèses sont assez bien vérifiées dans les tissus mous homogènes): $(a)$ monochromaticité, $(b)$ indépendance statistique des diffuseurs et indépendance statistique entre l'amplitude et la phase d'un même diffuseur (c) distribution uniforme des phases entre 0 et $2 \pi,(d)$ le nombre de diffuseurs qui contribuent au champ diffusé est grand. Le problème est alors identique à celui de la marche aléatoire dans plan complexe. On montre dans ces conditions que l'amplitude du signal suit une loi de Rayleigh. La valeur théorique du contraste en intensité pour une distribution de Rayleigh est égale à 1. La largeur de la fonction d'autocorrélation du speckle est déterminée par l'ouverture de la surface du transducteur. Dans la majeure partie des cas, la structure des tissus biologiques n'est qu'imparfaitement décrite par les hypothèses énumérées ci-dessus. La distribution de Rayleigh est un cas particulier d'une famille plus générale de distributions connues sous le nom de distributions de Rician. Ces distributions peuvent être obtenues dans le cas de milieux de propagation plus complexes: distribution semi-périodique de diffuseurs, présence de réflecteurs spéculaires, etc. C'est pourquoi certains auteurs s'attachent à caratériser la structure du tissu biologique à partir de l'analyse des propriétés statistiques du signal rf ou du signal vidéo. C'est le domaine de l'analyse de texture. La correspondance entre la texture du signal (d'où découle celle de l'image) et la "texture du tissu" (ou microstructure de $0.008 \mathrm{~mm}$ à $2 \mathrm{~mm}$ ) dépend fortement de la taille et de la distribution spatiale des diffuseurs du tissu par rapport à la longueur d'onde du signal émis. 


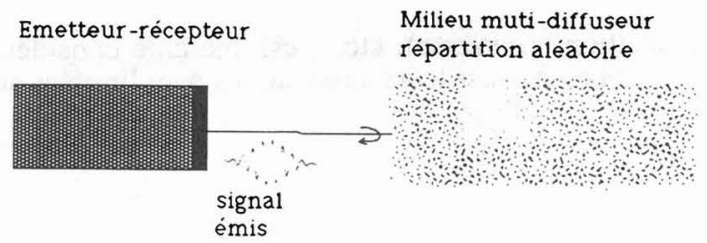

\section{SIGNAL RECU}

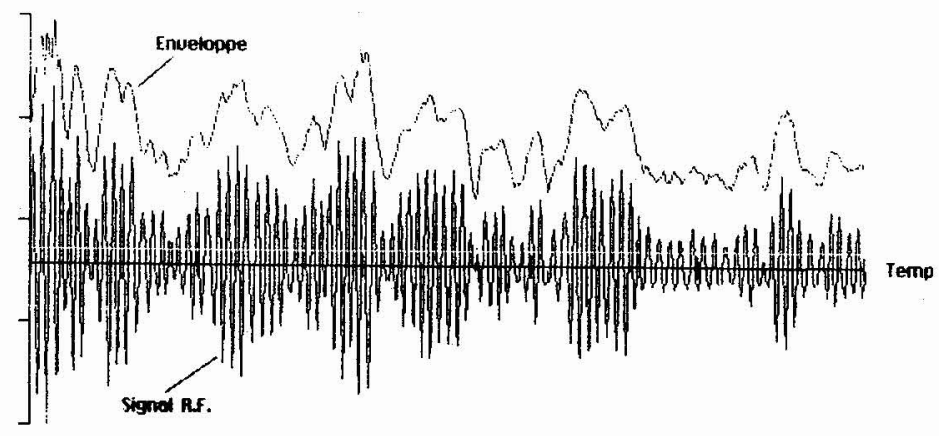

Fig.1.Illustration du signal radio-fréquence reçu par la sonde aprés interaction ultrasons-tissu (tracé inférieur). Enveloppe seule conservée pour la réalisation de l'image (traçé supérieur). Notion d'informations perdues.

La description statistique du signal échographique numérisé à haute fréquence, permet d'extraire plusieurs paramètres. Certains d'entre eux se corrèlent à des notions telles que : distance moyenne entre diffuseurs $[14,16]$, concentration des diffuseurs, rapport dans l'énergie réfléchie entre la part cohérente (réflexion spéculaire due aux structures de dimensions grandes devant la longueur d'onde) et la part incohérente (diffusion due aux structures de dimensions petites devant la longueur d'onde). Les approches précédentes ont été appliquées à la pathologie hépatique ou oculaire $[15,16]$.

D'autres paramètres, descriptifs de la statistique du premier et du deuxième ordre du signal, présentent un intérêt, non pas en tant que grandeur absolue, mais dans le cadre d'une analyse discriminante. Ils augmentent alors de manière significative et objective le taux de discrimination par ultrasons entre différentes pathologies $[2,9,10]$. Cependant, concernant cette dernière approche, les techniques développées donnent des paramètres qui sont système-dépendants. La base de donnée constituée localement par un équipement ne peut être implantée sur un autre système. Les recherches en cours visent à s'affranchir de ce problème. En ce sens, le deuxième domaine de la caractérisation tissulaire qui est basé sur l'extraction des paramètres acoustiques présente potentiellement l'intérêt de valeurs quantitatives absolues.

\subsection{Les paramètres acoustiques}

La détermination des paramètres acoustiques fait partie de l'échographie quantitative. Parmi les quatre "candidats" possibles figurent la célérité des ultrasons (vitesse de propagation), l'impédance acoustique des tissus, le coefficient de rétrodiffusion et l'atténuation des ultrasons.

Avant d'entrer dans le mode échographique, il convient de citer les approches tomographiques d'imagerie de transmission [3]. Leur intérêt est la réalisation d'images paramétriques. Sur une même coupe tomographique, Greenleaf présente plusieurs images : la cartographie de célérité, la cartographie d'atténuation, celle d'absorption et celle de diffusion. Ces images sont à notre sens tout à fait spectaculaires. Elles illustrent parfaitement le potentiel des ultrasons non encore utilisé en routine échographique. On ne peut s'empécher de comparer ces approches avec l'imagerie par résonance magnétique où la complémentarité d'images pondérées en T1 (temps de relaxation longitudinal), 
pondérées en T2 (temps de relaxation transverse), etc..., est une aide considérable au diagnostic. Il existe cependant une limite à ces approches car leurs applications sont limitées aux organes totalement
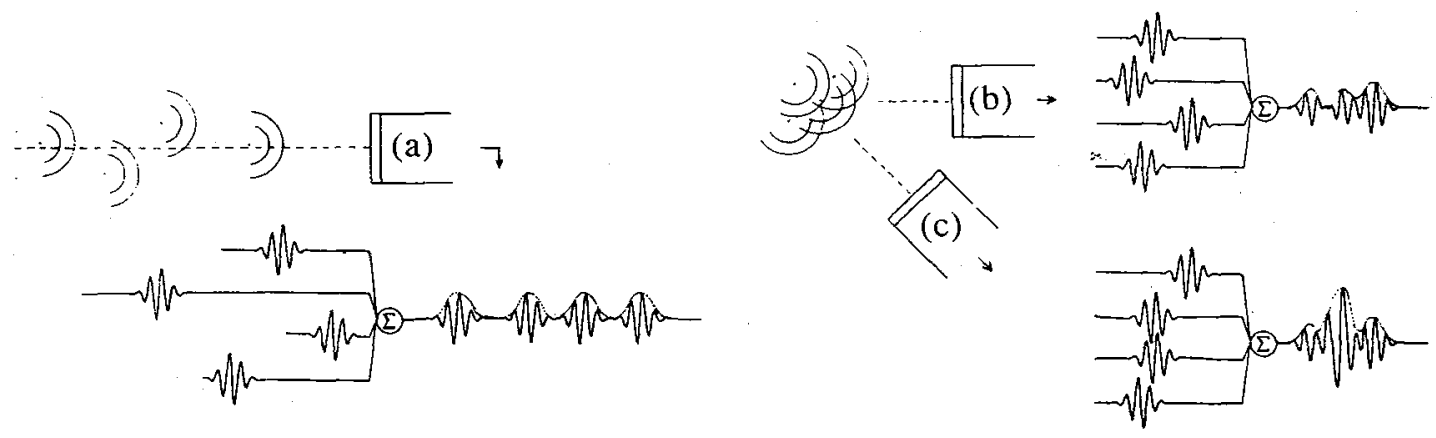

Fig.2.Sommation cohérente en un point de la surface du transducteur des ondelettes renvoyées par les diffuseurs aléatoirement distribués du tissu. (a) Densité de diffuseurs faible: pas d'interférences : cibles bien individualisées .(b) Densité de diffuseurs correspondant aux milieux biologiques : interférences, cibles non individualisées. (c) L'orientation du transducteur visant les mêmes diffuseurs est changée: modification du schéma d'interférences.

accessibles en transmission aux ultrasons, c'est-à-dire ne présentant pas d'interposition gazeuse ou osseuse. En pratique, elles ont été appliquées à la pathologie mammaire, qui est en soi un domaine clinique important. Nous allons maintenant nous focaliser dans la suite de cette présentation sur les techniques utilisables en mode échographique. Il convient néanmoins de garder à l'esprit la finalité suivante: la réalisation en mode de réflexion de ces mêmes images paramétriques.

\section{I.3.a Impédance acoustique:}

L'impédance acoustique " $Z$ " est le produit de la masse volumique par la vitesse de propagation dans le milieu. La rupture d'impédance acoustique entre deux interfaces conditionne le pourcentage de l'intensité ultrasonore réfléchie à ce niveau. Ainsi, dans le cas d'une onde d'incidence normale arrivant sur un interface plan séparant deux milieux d'impédances acoustiques $Z_{1}$ et $Z_{2}$, le rapport entre l'intensité incidente " $\mathrm{I}_{i}$ " et l'intensité réfléchie " $\mathrm{I}_{\mathrm{r}}$ " s'exprime :

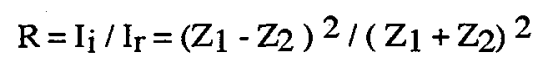

L'application de cette formule pour estimer l'impédance acoustique à partir du pourcentage d'intensité ultrasonore réfléchie à un interface est difficilement applicable in vivo. En effet, ce pourcentage est modifié par l'inclinaison du réflecteur par rapport au faisceau. Or il est difficile de controler ou de connaitre cet angle in vivo. Enfin, les réflecteurs de l'organisme sont loin d'être les réflecteurs-plan parfaits que suppose cette formule.

L'impédance peut également être considérée comme une fonction continue à une dimension. Dans ce cas, le coefficient de reflexion en fonction de la profondeur " $\mathrm{x}$ " devient :

$$
R(x)=0.5 d[\log Z(x)] / d x
$$

Les travaux dans ce domaine ont surtout été concentrés sur les méthodes de déconvolution [1]. Cette méthode aurait pu être utile pour l'étude de milieux à structures parallèles comme l'oeil. Il n'y a eu que peu d'application pratique in vivo. Il ne semble pas à l'heure actuelle que cette approche soit promettrice pour l'avenir de la caractérisation tissulaire. 


\section{I.3.b Célérité des ultrasons}

Dans l'imagerie conventionnelle, la célérité est supposée constante, voisine de celle de l'eau (1540 $\mathrm{m} / \mathrm{s}$ ). Dans l'hypothèse contraire, la simple connaissance du temps de vol des ultrasons ne permettrait pas de revenir à la notion de distance. De fait, les variations de célérité des ultrasons dans les tissus mous n'excèdent pas 10\%; la graisse présente les valeurs les plus basses aux environs de $1460 \mathrm{~m} / \mathrm{s}$ et le muscle les valeurs les plus élevées aux environs de $1700 \mathrm{~m} / \mathrm{s}$. Il faut noter que dans les conditions échographiques habituelles les fluctuations de célérité des différentes couches de tissu traversées sont responsables des fluctuations de phase du champ de pression. Ce phénomène est à l'origine de la dégradation des images échographiques et de nombreux auteurs se penchent actuellement sur les moyens de corriger ces fluctuations de célérité. Il apparait néanmoins que les variations de célérité, même faibles, sont assez significatives dans des contextes pathologiques. Parmi les différentes techniques proposées pour estimer cette vitesse, la première méthode introduite est due à Robinson [5]. Les ultrasons sont tout d'abord propagés dans l'eau, puis dans le tissu dont on cherche à estimer l'atténuation. Deux images d'une même cible identifiable dans la région d'intérêt (un vaisseau par exemple) sont effectuées sous deux directions différentes. En raison de la différence de célérité entre l'eau et le tissu, combinée à la différence d'angle d'incidence faisceau-interface pour chaque image, les deux images de la cible choisie n'apparaissent pas au même endroit (Fig.3). La connaissance de ce décalage, associée à la connaissance des angles d'incidences, permet de déterminer la célérité moyenne du tissu. La reproductibilité des mesures effectúes in vivo est de 1\%. Cette technique a été appliquée à la mesure de célérité dans des foies et des rates normaux et pathologiques [19].

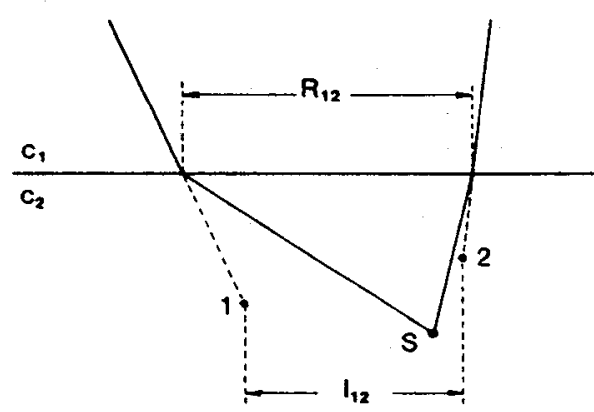

Fig.3.Principe d'estimation de la célérité [5]. $S$ est la position réelle de la cible, 1 et 2 sont ses images vues sous deux incidences différentes.après propagation dans un milieu de célérité c1 connue. La détermination de R12 et 112 permet d'estimer $\mathrm{c} 2$

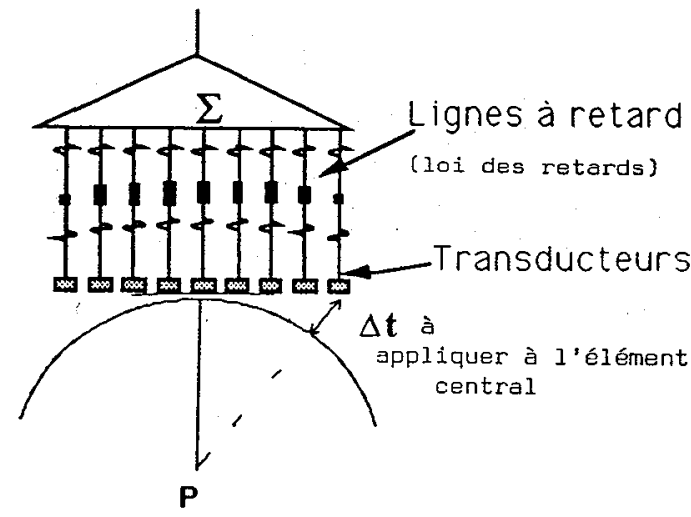

Fig.4.Estimation de la célérité à l'aide de barette à focalisation électronique : Dt est le retard à appliquer sur chaque voie, la focalisation optimale en $P$ permet de remonter à la célérité "créelle" du tissu.

D'autres méthodes ont été développées. On peut citer parmi celles-ci l'emploi de transducteurs à focalisation électronique avec grande ouverture. En effet la technique de focalisation électronique qui est nécessaire à la qualité de l'image peut également être utilisée pour estimer la célérité du milieu. La focalisation électronique en un point $P$ (émission et réception) est basée sur la compensation par des lignes à retard de la différence de temps de propagation $\mathrm{Dt}$ calculée pour chaque élément de la barette (Dt est représenté sur la figure 4). L'expression de Dt dépend de la célérité dans le milieu. Si la détermination de Dt est effectuée avec une valeur de "c" ne correspondant pas à la célérité réelle du milieu, il en résulte une focalisation imparfaite au point $\mathrm{P}$ avec diminution de la résolution. Ces considérations peuvent alors être utilisées pour estimer la célérité. Il suffit en effet de réaliser plusieurs images avec des valeurs itératives de "c". La valeur correcte (c'est à dire la célérité réelle du tissu) 
donnera la focalisation optimale avec une bonne résolution des contours et une bonne visibilité des détails. Enfin, le critère de focalisation optimale peut être subjectif (critère visuel d'appréciation de la qualité de l'image) ou objectif (critère quantitatif basé sur la notion d'entropie).

L'estimation de la vitesse de propagation des ultrasons semble très intéressante en raison de variations sensibles de ce paramètre dans les contextes pathologiques. Les différentes études menées in vitro et in vivo s'accordent pour conclure à une diminution de la célérité avec l'augmentation du contenu graisseux (foie) ainsi qu'avec l'augmentation du taux de fibrose (foie, rate). Dans un contexte de splenomégalie myéloïde, l'évolution de la célérité de la rate sous chimiothérapie concorde avec l'évolution de la biopsie médullaire [19].

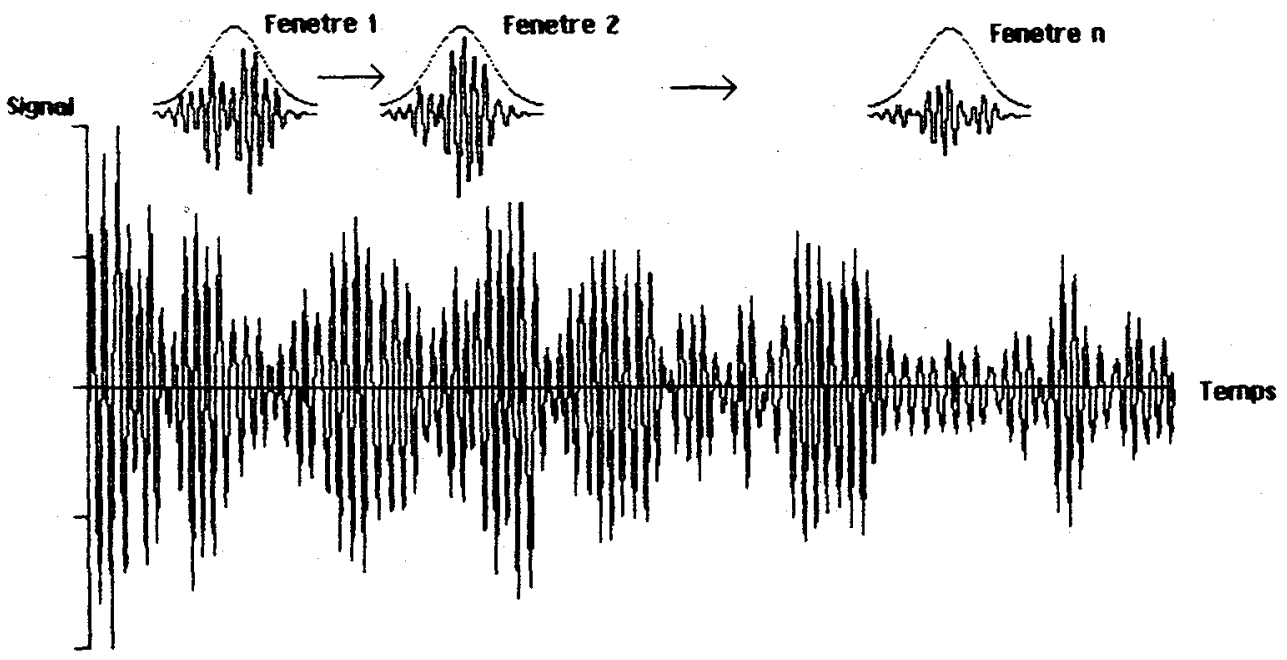

Fig.5.Principe d'obtention de la représentation temps-fréquence: déplacement d'une fenêtre temporelle avec taux de recouvrement le long du signal puis calcul du spectre de chaque portion de signal isolé par la fenêtre.

\section{I.3.c Coefficient de rétrodiffusion}

Le coefficient de rétrodiffusion se définit comme la section efficace de rétrodiffusion par unité d'angle solide et s'exprime en (cm.Stéradian) ${ }^{-1}$. Dans le myocarde d'un chien normal sa valeur est d'environ $10^{-1.9}$ (cm.Stéradian) ${ }^{-1}$ à $6.5 \mathrm{MHz}$ [4]. Sa variation avec la fréquence est rapide. Il est plus classique de caractériser la diffusion dans un volume d'intérêt par le coefficient intégral de rétrodiffusion. Ce paramètre est le rapport entre l'énergie diffusée par le volume (sélectionné par une porte temporelle) et l'énergie que réfléchit un réflecteur parfait positionné au même endroit du champ ultrasonore. Il s'exprime en dB. L'estimation est faite avec des transducteurs à large bande et ne permet pas d'apprécier la dépendance fréquentielle de la rétrodiffusion. Ces approches ont surtout été appliquées à la cardiologie [18].

L'estimation de ce coefficient en tant que valeur absolue est affectée par les tissus qui s'interposent entre le transducteur et le volume d'intérêt. En effet, l'atténuation qui en résulte, et qui n'est pas connue, peut biaiser le résultat. Cependant, dans le domaine de la cardiologie, il apparait que le paramètre présente des variations au cours du cycle cardiaque. L'amplitude de ces variations cycliques est diminuée en cas de nécrose ou d'ischémie, cette même amplitude pouvant permettre de suivre la 
récupération myocardique [18]. L'information de variation relative du paramètre est donc plus reproductible, plus informative et plus fiable que la valeur absolue. Enfin, cette approche permet d'obtenir une information assez localisée. La méthode a permis de déboucher sur des images bidimensionnelles du coefficient de rétrodiffusion integral obtenues en temps réel [25]. L'image modeB conventionnelle et celle du paramètre sont représentées simultanément. Nous devons à Miller des résultats extêmement convaincants dans le cadre des études myocardiques.

\section{3.d Atténuation ultrasonore}

L'atténuation des ultrasons résulte du mécanisme d'absorption (avec transformation sous forme de chaleur de l'énergie incidente) et du mécanisme de diffusion (avec une réémission de l'énergie dans toutes les directions de l'espace qui est à la base du signal échographique). C'est par ce paramètre acoustique que les tissus mous diffèrent le plus de l'eau. L'absorption y est en effet nettement plus importante en raison des macromolécules qu'ils contiennent. L'atténuation ultrasonore dépend de deux paramètres, d'une part de la profondeur de tissu traversé et d'autre part de la fréquence ultrasonore. Dans la gamme des fréquences médicales, la plupart des auteurs admettent que cette variation est quasilinéaire avec la fréquence (si l'on exprime l'atténuation en $\mathrm{dB}$ ). De cette double dépendance résulte un paramètre caractéristique du milieu qui s'exprime en $\mathrm{dB} / \mathrm{cm} . \mathrm{MHz}$. L'ordre de grandeur de ce paramètre dans les milieux biologiques est de $0.5 \mathrm{~dB} / \mathrm{cm} . \mathrm{MHz}$. Bien que l'augmentation de la fréquence améliore la résolution spatiale de l'image, la croissance proportionnelle de l'atténuation limite les applications à quelques $\mathrm{MHz}$ pour l'imagerie médicale. Cette limite se module en fonction de la profondeur d'exploration souhaitée. Enfin, si l'atténuation accompagnée de sa dépendance fréquentielle peut apparaitre comme une limite, elle peut aussi apparaitre comme un paramètre quantitatif d'intérêt dans le cadre de la caractérisation tissulaire.

Ce paramètre a trouvé beaucoup d'intérêt ces dernières années. Cet aspect recouvre notre propre domaine de recherche avec des travaux tout d'abord effectués dans le domaine de l'in vitro [6] puis dans celui de l'in vivo.

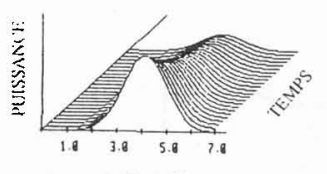

IRE.Q(E.XCE (MHz)

Atténuation $=0.75 \mathrm{~dB} / \mathrm{cm} \cdot \mathrm{MHz}$

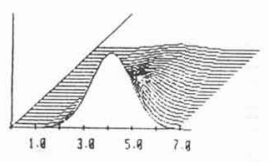

IREQLENCE, MHY,

Attenuation $=1.3 \mathrm{sB}, \mathrm{Or}, \mathrm{uHZ}_{\mathrm{HZ}}$

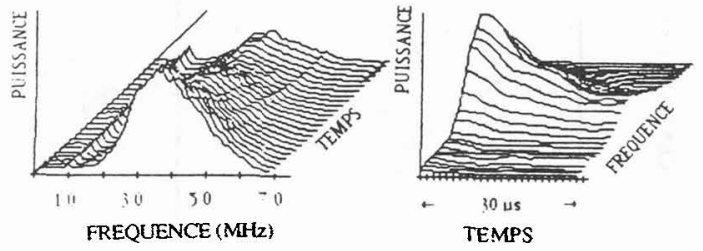

Fig.7.Evolution in vivo d'une représentation temps-fréquence acquise sur du tissu musculaire.
Fig.6.Simulation: aspect schématique des évolutions temps-fréquence en fonction de la profondeur pour deux milieux d'atténuations différentes.

\section{METHODOLOGIE D'ESTIMATION DE L'ATTENUATION}

\section{1 Représentation temps-fréquence du milieu}

Afin de conserver l'information fréquentielle, le signal acquis est le signal radio-fréquence (r.f.) et non le signal vidéo. La méthodologie est basée sur une analyse de Fourier glissante à courte période. Ce traitement permet d'obtenir la représentation temps-frequence du milieu. C'est à partir de cette représentation que peuvent être déterminée l'atténuation et sa dépendance fréquentielle. Deux algorithmes ont été développés. Le premier est l'algorithme du centroide, basé sur l'évolution de la fréquence "moyenne" en fonction de la profondeur de tissu. Le second est l'algorithme des bandes de fréquences qui permet d'obtenir la courbe d'atténuation en fonction de la fréquence. Un échographe 
conventionnel est utilisé pour le repérage anatomique de la région dans laquelle on souhaite estimer l'atténuation. Les signaux réfléchis par cette région sont prélevés directement à la sortie de la sonde échographique après une simple pré-amplification. Dans nos expériences, la fréquence centrale de la sonde est de $5 \mathrm{MHz}$ et la fréquence d'échantillonnage est de $25 \mathrm{MHz}$. La représentation tempsfréquence est obtenue en déplaçant sur le signal une fenêtre temporelle avec un certain pourcentage de recouvrement (Fig. 5). Le spectre de puissance est calculé dans chaque fenêtre, pondérée par une fonction de type Hanning. La représentation de l'ensemble de ces spectres en fonction de la profondeur est la représentation temps fréquence tridimensionnelle de cette ligne échographique.

Quel est l'aspect attendu de la représentation temps-fréquence d'un milieu atténuant? Deux illustrations de deux milieux d'atténuations différentes sont données sur la figure 6 . On observe la décroissance de l'intensité de chaque fréquence en fonction de la profondeur (ou temps de propagation). On observe une décroissance plus marquée pour le milieu présentant l'atténuation la plus élevée. Sans rentrer pour l'instant dans les détails et problèmes relatifs à son mode d'obtention, nous pouvons d'emblée illustrer une représentation temps-fréquence obtenue in vivo. L'aspect est proche de l'aspect théorique attendu (Fig. 8). Pour estimer l'atténuation ultrasonore à partir de ces données, deux algorithmes sont présentés.

\section{II.2. Algorithmes}

L'atténuation que subit chaque fréquence du spectre est plus marquée pour les hautes fréquences spectrales que pour les basses fréquences. Ainsi, si l'on considère le centre de gravité de ce spectre (centroide), il aura tendance à se décaler vers les basses fréquences au fur et à mesure que l'on pénètre dans le milieu. Plus la dépendance de l'atténuation avec la fréquence est élevée, plus cette chute est rapide. Dans le cas particulier d'un spectre gaussien et d'une atténuation linéaire avec la fréquence (s'exprimant alors en $\mathrm{dB} / \mathrm{cm} . \mathrm{MHz}$ ) la décoissance du centroide avec la profondeur est une droite de pente proportionnelle à cette atténuation [7]. Précisons que le calcul passe par la détermination des moments spectraux d'ordre 0,1 et 2 . L'hypothèse d'un spectre gaussien n'est pas une nécessité, la formulation est simplement un peu moins simple. Une variation conforme à la théorie peut être appréhendée in vivo. Une illustration en est donnée figure 8.

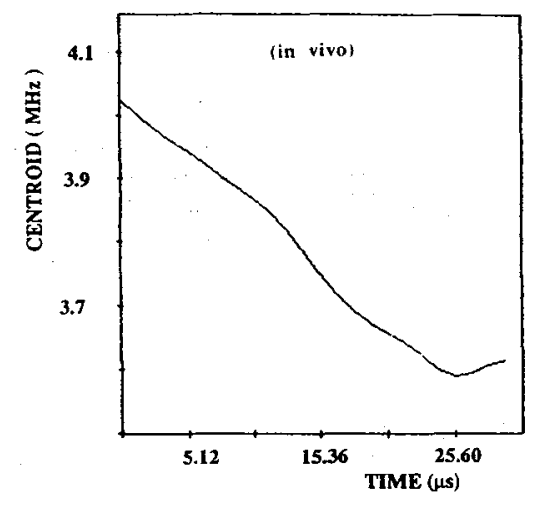

Fig.8.Evolution in vivo du centroid musculaire conforme à la variation linéaire attendue. La pente l'évolution du centroîde est directement proportionnelle à l'atténuation $(\mathrm{dB} / \mathrm{cm} . \mathrm{MHz})$ du milieu.

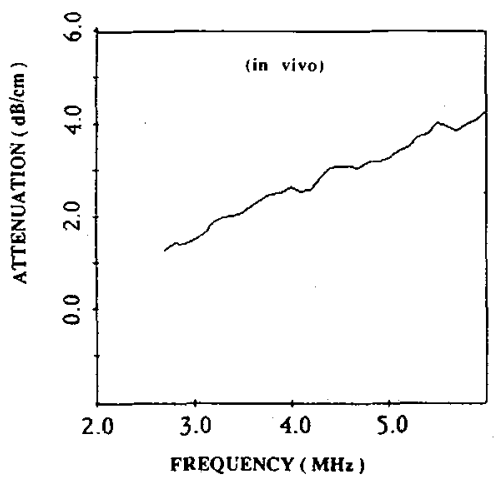

Fig.9.Exemple de courbe d'atténuation obtenue in vivo sur du tissu musculaire par l'algorithme de des bandes de fréquences.

Comme le précédent algorithme, l'algorithme des bandes de fréquences est directement appliqué à la représentation temps-fréquence. La décoissance du logarithme décimal de l'intensité avec la profondeur est calculée pour chaque fréquence et permet d'estimer l'atténuation correspondante en $\mathrm{dB} / \mathrm{cm}$. On obtient ainsi la courbe d'atténuation de fonction de la fréquence, ce qui permet de vérifier la dépendance linéaire de l'atténuation avec la fréquence puis d'estimer la pente de cette courbe (exprimée en $\mathrm{dB} / \mathrm{cm} . \mathrm{MHz}$ ). Les aspects observés in vivo confortent les hypothèses de départ (Fig. 9). 
Ces deux algorithmes peuvent être comparés. Dans les deux cas, la réprésentation temps-fréquence tridimensionnelle est comprimée en deux dimensions. Dans l'algorithme du centroide, l'ensemble de la répartition du contenu spectral est condensé dans la notion de centroide. L'inconvénient en est l'hypothèse implicite de linéarité de l'atténuation avec la fréquence; l'avantage en est la conservation de l'évolution avec la profondeur permettant de détecter la survenue d'éventuels accidents (pouvant notamment être dus à des réflecteurs speculaires [12]) - Dans l'algorithme des bandes de fréquences, c'est l'évolution en fonction de la distance qui est comprimée. L'inconvénient est de ne pouvoir détecter les accidents survenant en fonction de la profondeur, l'avantage est de conserver l'évolution avec la fréquence et de controler l'hypothèse de linéarité. Au total, ces deux approches se complètent pour décripter la représentation temps-fréquence.

D'autres algorithmes peuvent être trouvés dans la littérature : algorithme de comptage des passages à zéro du signal pour déterminer la fréquence moyenne, algorithme basé sur la détection des pics d'enveloppe [26], algorithme utilisant des techniques proches des techniques doppler [31].

\section{II.3 Problèmes posés}

L'application directe des considérations précédentes se heurte à quelques problèmes qu'il fallut résoudre. Le premier problème est lié à la variance de l'estimation de l'atténuation. La nature stochastique du signal échographique imposée elle-même par les propriétés statistiques des tissus mous (Fig. 2) a pour conséquence le fait suivant: la pente de l' atténuation estimée est une variable aléatoire. En modélisant le tissu comme un mileu multi-diffuseurs de distribution aléatoire, il est possible d'obtenir la formulation des lois statistiques de premier ordre concernant $l^{\prime}$ atténuation. Ces considérations aboutissent à l'importante conclusion suivante: la variance sur la pente de l' atténuation varie en (WD) ${ }^{-3}$, où $\mathrm{W}$ représente la largeur de bande de la sonde, $\mathrm{D} \mathrm{l}^{\prime}$ épaisseur de tissu exploré. Ce résultat met en valeur les deux paramètres dont l' optimisation est nécessaire pour une meilleure convergence statistique de la grandeur estimée [23]. La réduction de la variance passe par des techniques de moyennage. Le moyennage spatial a été principalement utilisé jusqu'alors. D'autres techniques de moyennage, basées sur l'introduction d'écrans de phase aléatoire semblent pleines d'avenir [31]. Ces considérations expliquent qu'au travers de la littérature les principales applications cliniques présentées concernent des pathologies diffuses.

Le second problème est du aux échos spéculaires par la perturbation qu'ils apportent sur les distributions d'énergie temps-fréquence [12]. L'hétérogénéité d'un tissu est responsable de la noninvariance du filtre de diffusion et d'un biais sur l'estimation de l'atténuation. Enfin, le dernier problème, dont la compréhension a beaucoup fait avancer l'estimation in vivo de l'atténuation est celui des effets de diffraction mis en avant par Fink [8]. Les effets de diffraction sont causés par les variations (en fonction de la profondeur) des temps relatifs de propagation de l'onde provenant des différents points de l'ouverture émettrice: par exemple, un transducteur plan excité en mode piston rayonne à la fois une onde plane dans l'ombre géométrique du transducteur et une onde de bord émise par les bords du disque. La pression transitoire observée au point d'observation est le résultat de l'interférence entre ces deux ondes. Lorsque des transducteurs de taille finie sont excités par des impulsions de courte durée, les fronts d'ondes de pression transitoires et leur spectre en fréquence dependent du point d'observation. Par conséquent la diffraction agit comme un filtre qui varie avec le point d'observation. En mode échographique impulsionnel, la relation existant entre le temps de vol de l'écho et la profondeur à laquelle se trouve la cible diffusante nous permet de dire que la diffraction agit comme un filtre variable dans le temps. De ce fait ce filtre vient ajouter ses effets à celui de l'atténuation et peut être responsable d'un biais sur la mesure de l'atténuation. Une correction de diffraction est nécessaire. .

Sur les définitions précédentes, un système permettant l'estimation en temps réel de l'atténuation a été mis au point. Son originalité est basée sur une unité performante de calcul des spectres de puissance. Ce système est une ouverture sur les études cliniques.

\section{APPLICATIONS CLINIQUES DE L'ESTIMATION DE L'ATTENUATION}

\section{III.1 Pathologie musculaire : myopathie de Duchenne}

La détection des transmettrices de la myopathie de Duchenne a suscité de nombreuses recherches utilisant des méthodes chimiques ou physiques. La plus ancienne a été le dosage de l'aldolase sérique, 
suivie par le dosage de créatine-kinase mais cette dernière ne détecte qu'environ $70 \%$ des porteuses génétiques. A côté des méthodes du génie génétique utilisables actuellement pour le diagnostic prénatal lorsque les familles sont informatives [22] nous avons appliqué les techniques d'échographie quantitative par mesure du coefficient d'atténuation du signal ultrasonore sur le tissu musculaire.

Nous avons effectué des mesures préliminaires sur des garçons myopathes, puis sur un groupe témoin de 30 femmes sans anomalies musculaires et à CK normale, puis sur un goupe de 45 transmettrices sûres de la myopathie de Duchenne dont dix-neuf classées uniquement suivant les critères génétiques habituels. Les enfants présentent systématiquement des atténuations trés élevées par rapport à la normale. La différence $d^{\prime}$ atténuation moyenne entre la population de femmes témoins (30) et la population de porteuses (45) est significativement différente ( au risque de 1\%) (Fig. 10).

Une analyse quantitative de l'atténuation des ultrasons lorsqu'elle est couplée à un dosage de créatine-kinase augmente considérablement la probabilité de détection [13]. Si les deux méthodes ne sont pas corrélées, cela provient, selon toute vraisemblance, du fait qu'elles n'explorent pas les mêmes lésions. La perméabilité anormale des membranes cellulaires rend compte de la créatine-kinasémie. L'épaisseur très faible de ces membranes les rend impropres à la détection par les ultrasons. En revanche, s'il existe dans le muscle des porteuses de petits îlots dégénératifs, comportant des infiltrations graisseuses, ces derniers constituent autant de cibles diffusantes susceptibles de justifier l'augmentation observée du coefficient d' atténuation.

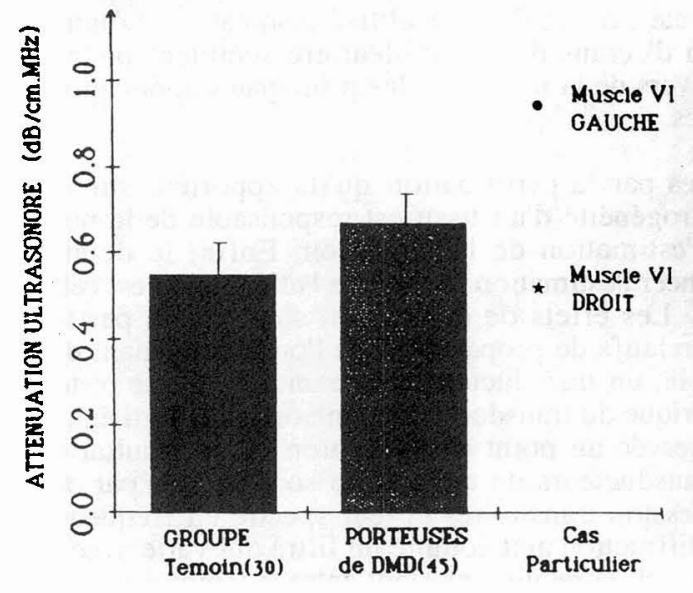

Fig.10.Attenuation moyenne d'une population témoin et d'une population de porteuses certaines de la myopathie de Duchenne. Présentation d'un cas particulier de porteuse asymptomatique avec nette asymétrie de l'atténuation musculaire des vastes internes.

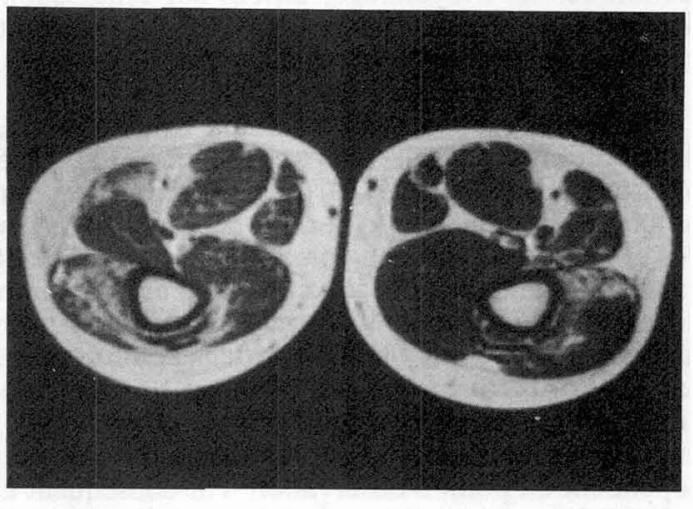

Fig.11.Images de coupes musculaires obtenues par résonnance magnétique nucléairechez une porteuse possible (atténuation normale à droite, élevée à gauche). Images de coupes musculaires montrant une trés nette asymétrie de l'infiltration lipidique L'infiltration lipidique est beaucoup plus marquée à gauche. 
Sur cette dernière hypothèse, nous avons effectué des corrélations avec l'imagerie par résonance magnétique nucléaire, en raison des possibilités de contraste élevé entre muscle et graisse. Quelques cas particuliers ont été étudiés. Nous présentons ici le cas le plus intéressant. Il s'agissait d'une porteuse possible (soeur $d^{\prime}$ un garçon myopathe) chez laquelle nous avions enregistré une différence très marquée entre les atténuations mesurées à droite et à gauche (Fig. 10: cas particulier). Les images IRM (fig. 11) en coupe de muscles de la cuisse valident l' asymétrie observée en montrant à gauche une infiltration très prononcée par la graisse, alors que les images des muscles à droite étaient normales. Ainsi l'hypothèse selon laquelle l'augmentation de l'atténuation des ultrasons est corrélée à l'infiltration lipidique des muscles des femmes porteuses de la myopathie de Duchenne se trouve confirmée. Ces observations sont particulièrement intéressantes, elles valident les techniques d'estimation de l'atténuation, leur intérêt et leur signification clinique.

\section{2 Atténuation et glande mammaire}

Nous avons étudié l'apport potentiel de l'atténuation ultrasonore comme index quantitatif de la glande mammaire pour la caractérisation et le suivi des mastopathies bénignes. La première étape fut l'investigation d'une population témoin incluant plus de 100 sujets. Un premier sous-groupe de 50 témoins, de distribution d'âge uniforme (19 à 65 ans) met en évidence une assez grande dispersion de l'atténuation ultrasonore: $1.68 \pm 0.48 \mathrm{~dB} / \mathrm{cm}$. MHz. La corrélation avec des facteurs physiologiques (durée de vie génitale, grossesses, phase du cycle, antécédent mammaire personnel ou familial, etc.) a été estimée par une régression multi-linéaire. Il se dégage principalement une corrélation entre l'atténuation et la durée de vie génitale, paramètre le plus significatif pour expliquer la variation. Un second sous-groupe de 50 sujets de même âge environ complète cette approche. Enfin, une étude préliminaire inclue 10 cas pathologiques de mastopathies bénignes. Le paramètre type (normal ou pathologique) qui les discrimine dans la régression multi-linéaire apparaît alors comme le paramètre le plus significatif. Ce résultat préliminaire semble encourageant.

Compte-tenu des observations précédentes, l'hypothèse la plus vraisemblable permettant d'interpréter la modification de l'atténuation avec la durée de vie génitale est de relier cette modification à l'involution lipidique de la glande mammaire.

\section{3 Atténuation et caractérisation tissulaire}

Bien que les publications cliniques dans ce domaine soient limitées, l'organe le plus exploré a été le foie. Les raisons en sont : la taille de l'organe, l'accessibilité échographique, la nature diffuse de l'atteinte dans de nombreuses pathologies hépatiques. Il ne semble pas qu'il faille regarder l'atténuation comme un paramètre de discrimination entre les différentes pathologies. En effet cirrhose comme hépatite recouvrent en elles-même une grande variété de modifications histologiques. L'atténuation reflète mieux la nature de la transformation du parenchyme hépatique. Ainsi, l'augmentation de l'atténuation se corrèle avec le degré de stéatose [17]. Ces conclusions concordent avec nos propres observations concernant le role de l'infiltration lipidique d'un tissu sur l'atténuation. La corrélation est beaucoup plus faible avec le degré de fibrose [27]. Enfin, ce paramètre est également susceptible de se modifier en fonction du taux de glycogène [28].

\section{CONCLUSION}

Le but de la caratérisation tissulaire est d'apporter des informations sur les tissus que l'image conventionnelle ne présente pas. Les grandes voies qui se dégagent sont: l'analyse de texture, la détermination des paramètres de célérité, de rétrodiffusion et d'atténuation. L'aspect quantitatif est intéressant pour deux raisons. D'une part il est une aide au diagnostic et pallie à l'aspect opérateurdépendant de la routine échographique. D'autre part l'inocuité, la reproductibilité des estimations quantitatives en font des techniques très bien adaptées au suivi des pathologies et à l'évolution sous thérapeutique. Nous avons ainsi montré que l'atténuation des ultrasons par le muscle reflète l'infiltration par de la graisse, elle-même liée à la dégénérescence musculaire. La corrélation avec les techniques d'IRM valide de manière certaines les informations cliniques contenues dans le paramètre atténuation. Il est légitime de penser que la mesure quantitative de ce paramètre peut servir à la surveillance de l'évolution de l'atteinte musculaire chez les garçons atteints de myopathie de Duchenne et s'inclure dans la série des tests d'évaluation thérapeutique qui seront suscités dans un court ou moyen terme par les récentes découvertes sur le gène de la myopathie de Duchenne. Dans la même optique, la normalisation de la célérité splénique atteste de la réduction du taux de fibrose, l'évolution de la célérité hépatique suit 
la réduction du degré de stéatose et la récupération de l'amplitude cyclique de variation du coefficient de rétrodiffusion du myocarde est en faveur d'une reperfusion. Par ailleurs, l'ensemble des travaux publié sur la caractérisation myocardique est exemplaire de l'intérêt potentiel des ultrasons. Ils attestent de l'ensemble des informations sur les tissus que contiennent les signaux réfléchis et que l'image ne représente pas.

Il faut également insister sur le fait que la détermination multiparamètres est en elle-même un centre d'intérêt. Il a été monté par exemple que l'atténuation ultrasonore se majorait avec le taux d'infiltration lipidique du tissu mais que le degré de fibrose l'affectait moins. Il a été montré par ailleurs que la célérité diminuait avec l'augmentation de l'infiltration graisseuse ainsi qu'avec le taux de fibrose. On concoit aisément l'intérêt d'une détermination conjuguée de ces deux paramètres pour une approche de type discriminante.

Si les paramètres peuvent bien sur être estimés de manière globale, il faut également envisager la réalisation simultanée d'images paramétriques. De telles images ont déjà été obtenues en transmission. Ces images sont impressionnantes et convaincantes sur l'apport potentiel encore non exploité en routine des ultrasons. Cependant, dans l'imagerie de transmission, leur application est limitee au sein. Concernant le mode échographique, les images de paramètres de texture ou de coefficient de rétrodiffusion ont déjà été présentées. Cependant, si l'on est maintenant en mesure d'estimer l'atténuation et la célérité sous la forme d' un index tissulaire global, paramètre fiable, reproductible et quantitatif, il persiste jusqu'alors une limite concernant la faible résolution spatiale de ces estimations. Cette limite explique que le champ des applications ait porté sur les pathologies diffuses. Elle justifie le développement de nouveau axes de recherche pour améliorer la résolution Concernant l'atténuation des ultrasons, des améliorations notables doivent être obtenues par l'utilisation de nouvelles sondes : soit des sondes hautes haute-fréquence, soit des sondes incohérentes ou des sondes sans effet de diffraction.

La caractérisation tissulaire et son corrolaire, l'échographie quantitative, abordent donc véritablement le stade de l'évaluation clinique. En effet, les expériences effectuées in vitro datent de quelques dizaines d'années et la possibilité d'une extension au domaine de l'in vivo restait une question en suspend. Il aura fallu que des travaux fondamentaux et théoriques elucident quelque peu ces mécanismes complexes d'interaction des ultrasons avec les tissus. Il aura fallu que cette compréhension aboutisse à des algorithmes permettant d'évaluer de manière significative et reproductible les paramètres acoustiques d'un milieu. Il aura fallu également le développement de nouvelles technologies d'acquisition et de traitement des signaux. Cette étape était nécessaire, elle est encore insuffisante pour crier victoire. Dans ce stade du développement, les différentes techniques ne sont pas encore implantées sur des systèmes de routine ou des systèmes commerciaux. L'évolution prochaine de ces techniques devrait apporter une réponse définitive sur leur valeur clinique et décider de leur implantation sur des systèmes de routine.

\section{BIBLIOGRAPHIE}

[1] HERMENT, A., PERONNEAU, P., VAYSE, M. , A vew method of obtaining an acoustic impedance profile for characterization of tissues structures, Ultrasound in Med. and Biol., 5,321332 (1979)

[2] LERSKI R.A., MORLEY P., MILLS P.R. and WATKINSON G., Texture Analysis of UItrasonic Signals - A Comparison of the Use of Radiofrequency and Demodulated A-SCAN DATA, Ultrasonic Imaging 3, pp 369 - 377 (1981)

[3] GREENLEAF, J.F., Computerized transmission tomography, Methods of Experiment Physics Ultrasound, P.D. Edmonds, ed, 19, 563 - 589, (Academic Press, New-York, 1981).

[4] O'DONNEL, M., MILLER, J.G., Quantitative broadband ultrasonic backscatter: an approach to non-destructive evaluation in acoustically inhomogeneous materials, J. Appl. Phys., 52, 1056 (1981)

[5] ROBINSON D. E., CHEN, F., WILSON, L.S., Measurement of velocity of propagation from ultrasonic pulse echo data, Ultrasound in Med. and Biol., 8, 413-420 (1982) 
[6] BERGER G. , and coll. , Role Played by cellular organisation and cohesion in the interaction of ultrasound with soft tissues, Acoustical lmaging. Vol 10 (Ed. P. Alais and A.F. Mfetherell) Plenum Press New-York p. 253 - 264 (1982).

[7] FINK,M., HOTTIER, F., CARDOSO, J.F., Ultrasonic signal processing for in vivo attenuation measurement: short time Fourier analysis, Ultrasonic Imaging 5, 117 - 135 (1983)

[8] CARDOSO, J.,F., FINK,M., Diffraction Correction in Pulse Echo Attenuation Measurement, IEEE Ultrasonic Symposium Proceedings, IEEE CAT 83 CH 1947-1, pp 841 - 846, (1983)

[9] FINETTE, S., BLEIER, A., SWINDELL, W., and HARBER, K., Breast tissue classification using diagnostic ultrasound and pattern recognition techniques : Il Experimental results, Ultrasonic Imaging 5, 71 - 86, (1983).

[10] FINETTE, S., BLEIER, A., and SWINDELL, W., Breast tissue classification using diagnostic ultrasound and pattern recognition techniques : I methods of pattern recognition, Ultrasonic Imaging 5, 55 - 70, (1983).

[11] WILSON, L.S., ROBINSON, D.E. and DOUST, B.D., Frequency domain processing for ultrasonic attenuation measurement in liver, Ultrasonic Imaging 6, 278- 292 (1984).

[12] LAUGIER P., BERGER G., FINK M., PERRIN J., Specular Reflector noise: Effect and Correction for In Vivo Attenuation Estimation, Ultrasonic Imaging 7, pp. 277-292 ( 1985 )

[13] SCHAPIRA G., LAUGIER P., ROCHETTE J., BERGER G., PERRIN J., Detection of Muscular Dystrophy Carriers: Quantitative Echography and Creatine-Kinasemia, Human Genetics 75, pp $19-23$ (1986)

[14] WAGNER R.F., INSANA M.F., BROWN D.G., Unified approch to the detection and classification of speckle texture in diagnostic ultrasound, Optical Engineering Vol 25, pp 738 742 (1986)

[15] LIZZI F.L., OSTROMOGILSKY M., FELEPPA E.J., RORKE M.C., and YAREMKO M.M., Relationship of Ultrasonic Spectral Parameters to Features of Tissue Microstructure, EEE Trans. on Ultr. Ferr. and Freq. Cont. Vol UFFC-33, (1986)

[16] INSANA M.F., WAGNER R.F., GARRA B.S., BROWN D.G., SHAWKER T.H., Analysis of ultrasound image texture via generalized Rician statistics, Optical Engineering 25, pp 743-748 (1986)

[17] TAYLOR, K.J., RIELY, C.A., HAMMERS, L., FLAX, S., WELTIN, G., GARSIA-TSAO, G., CONN, H.O., KUC, R., BARWICK, K.B., Quantitative US attenuation in normal liver and patients with diffuse liver disease : Importance of Fat, Radiology 160, 65 - 75, (1986).

[18] WICKLINE, S.A., THOMAS III, L.J., MILLER, J.G., SOBEL, B.E., PEREZ, J.E., Sensitive detection of the effects of reperfusion on myocardium by ultrasonic tissue characterization with integrated backscatter, Circulation, 74, 389-400 (1986).

[19] WILSON,L.S.,ROBINSIN,D.E.,GRIFFITHS,K.A.,MANOHARAN,A.,DOUST,B.D., Evaluation of ultrasonic attenuation in diffuse diseases of spleen and liver, Ultrasonic Imaging 9 , 236-247 (1987).

[20]CHEN,C.F,ROBINSON,D.E.,WILSON,L.S.,GRIFFITHS,K.A.,MANOHARAN,A.,DOUST,B .D., CLinical Sound Speed Measurements in Liver and Spleen In Vivo, Ultrasonic Imaging 9 , 221-235 (1987).

[21] LAUGIER P., BERGER G., FINK M., PERRIN J., Diffraction correction for focused transducers in attenuation measurements in vivo, Ultrasonic Imaging 9, 248-259 (1987)

[22] HODGSON,S.,WALKER,A.,COLE,C.,HART,K.,JOHNSON,L.,HECKMATT,J. DUBOWITZ,V.,BOBROW,M., The application of linkage analysis to genetic counselling in families with Duchenne or Becker muscular dystrophy, J. Med. Genetics 24,152-159 (1987)

[23] BERGER G., LAUGIER P., FINK M., PERRIN J., Optimal precision in ultrasound attenuation estimation and application to the Duchenne muscular dystrophy carriers detection, Ultrasonic Imaging 9, 1 - 17(1987)

[24] BERGER G., LAUGIER P., FINK M., PERRIN J., Attenuation as an additive clinical indicator, Ultrasonic tissue characterization and ultrasonic imaging 6 (ED. J.M. Thijssen and G. Berger) p. $101-112(1987)$

[25] VERED, Z., BARZILAI, B., MOHR, G.A., THOMAS IH, L.J.,PEREZ, J.E., SOBEL, B.E.,MILLER, J.G., Real-Time Two-dimensional imaging based on radio-frequency-derived integrated backscatter in normal volunteers and patients, Ultrasonic Imaging 9, 51 (1987)

[26] HE,P. , On the estimation of acoustic attenuation coefficient from peaks of echo envelope, $J$. Acoust. Soc. Am 83 (5), 1919-1926 (1988)

[27] LIN,T., OPHIR, J., POTTER G., Correlation of ultrasonic attenuation with pathologic fat and fibrosis in liver disease, Ultrasound in Med. and Biol. 14 (8) 729-734 (1988) 
[28] PARKER,K.J., TUTHILL, T.A., BAGGS R.B., The role of glycogen and phosphate in ultrasonic attenuation of liver, J. Acoust. Soc. Ame. 83(1) 374-378 (1988)

[29] LYMBERIS, A., HERMENT A, DEMOMENT G., FRIC C., Estimation of frequency-dependent attenuation based on parametric spectral analysis and correlation lags of $\mathrm{rf}$ echograms signals, Ultrasonic Imaging 11, 138 (1989)

[30] BERGER, G., LAUGIER, P. THALABARD, J.C., PERRIN, J., Global breast attenuation : control group and benign breast diseases, Ultrasonic Imaging 12, 47-57 (1990)

[31] LAUGIER, P., FINK, M., ABOUELKARAM, S., The random phase transducer : a new technique for incoherent processing - Experimental results, IEEE Trans. on Ultrasonics, ferroelectrics and frequency control, Vol. 37, 2, p. 70-78 (1990) 